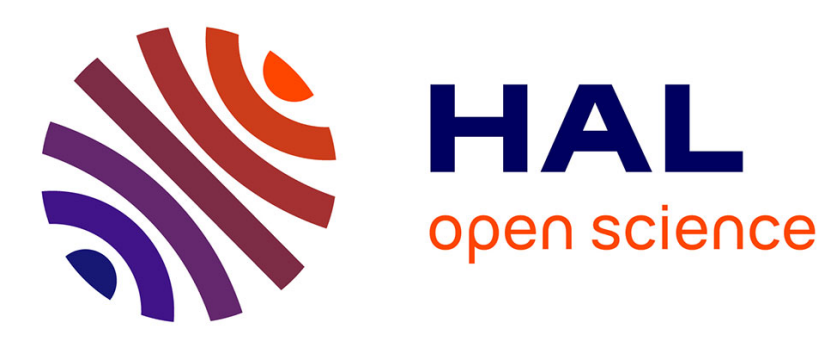

\title{
Mechanical modeling of indentation-induced densification in amorphous silica
}

Guillaume Kermouche, Etienne Barthel, D. Vandembroucq, Philippe Dubujet

\section{To cite this version:}

Guillaume Kermouche, Etienne Barthel, D. Vandembroucq, Philippe Dubujet. Mechanical modeling of indentation-induced densification in amorphous silica. Acta Materialia, 2008, 56 (13), pp.3222-3228. hal-00260324

\section{HAL Id: hal-00260324 \\ https://hal.science/hal-00260324}

Submitted on 3 Mar 2008

HAL is a multi-disciplinary open access archive for the deposit and dissemination of scientific research documents, whether they are published or not. The documents may come from teaching and research institutions in France or abroad, or from public or private research centers.
L'archive ouverte pluridisciplinaire HAL, est destinée au dépôt et à la diffusion de documents scientifiques de niveau recherche, publiés ou non, émanant des établissements d'enseignement et de recherche français ou étrangers, des laboratoires publics ou privés. 


\title{
Mechanical modelling of indentation-induced densification in amorphous silica
}

\author{
G. Kermouche ${ }^{\mathrm{a}, *}$, E. Barthel ${ }^{\mathrm{b}}$, D. Vandembroucq ${ }^{\mathrm{b}}$, \\ Ph. Dubujet ${ }^{\mathrm{a}}$ \\ ${ }^{a}$ Ecole Nationale d'Ingénieurs de Saint-Etienne, Laboratoire de Tribologie et \\ Dynamique des Systèmes, UMR 5513 CNRS/ECL/ENISE, \\ 58 rue J. Parot 42023 Saint-Etienne Cedex 2,France \\ ${ }^{\mathrm{b}}$ Surface du Verre et Interfaces, CNRS/Saint-Gobain, UMR 125, \\ 39, Quai Lucien Lefranc, 93303 Aubervilliers Cedex, France
}

\begin{abstract}
Though brittle, silicate glasses undergo plastic deformation at the microscopic scale. Mechanical contact and indentation are the most common situations of interest. The plasticity of glasses is characterized not only by shear flow but also by a permanent densification process. After indentation, densification can locally reach $20 \%$ in a pure silica glass. In this paper, a new constitutive model, derived from experimental observations is presented to account for the plasticity of fused silica. The use of nanoindentation tests to identify the plastic behavior of amorphous silica is discussed. A set of material properties is determined by comparing experimental load/displacement indentation curves to the results of finite element simulations. The numerical results show good agreement with recent experimental indentationinduced densification maps obtained by Perriot et al. [J. Am. Ceram. Soc., 89:596$601,2006]$.
\end{abstract}


Key words: Pressure dependent plasticity, densification maps, Nanoindentation, Finite element method

\section{Introduction}

Though primarily brittle [1], silicate glasses can undergo plastic deformation at the micron scale. his can occur under contact loading [2] or in the close vicinity of a crack tip. In that respect glasses do not differ from standard materials, except that the characteristic length scale below which volume deformation (plasticity) is energetically more favorable than surface creation (crack propagation) is smaller [3]. Although known for half a century [4] and of primary interest for many micron-scale applications, the plasticity of silicate glasses has up to now remained little studied [5-8].

Standard dislocation-based mechanisms of crystalline solids is of no direct use to explain the plasticity of silicate glasses because they are amorphous. Instead, according to the dominant scenario, plastic deformation of glasses seems to result from a succession of localized structural rearrangements $[9,10]$. On the plasticity of amorphous materials and more specifically of metallic glasses, see for instance the recent review of Schuh[11].

Moreover, plasticity of silicate glasses is characterized by one more specific feature. Indeed, it appears that a more or less significant phenomenon of permanent densification [12] takes place in addition to the isochoric plastic strain classically observed in metallic materials. This characteristic leads to

* Corresponding author. Tel.:+33(0)4.77.43.75.31; Fax:+33(0)4.77.43.75.39

Email address: guillaume.kermouche@enise.fr (G. Kermouche). 
a distinction between normal glasses and anomalous glasses: normal glasses exhibit little densification while anomalous glasses exhibit large densification as well as a different indentation fracture pattern [13]. Fused silica, the most anomalous silicate glass of industrial interest, can achieve a significant $20 \%$ densification [5].

The lack of studies on this subject is directly related to the brittle nature of the material and the necessity to resort to mechanical measurements at a micron scale. Progress has been obtained thanks to the recent developments in instrumented nanoindentation techniques $[14,15]$. Nanoindentation tests are micromechanical tests where the load applied to a tip is continuously measured as a function of the penetration depth. Recently it has been successfully used to extract material properties such as hardness or elastic modulus of very thin devices [16-18]. Nevertheless, a single load-penetration curve does not provide a unique set of material properties for even moderately complex materials such as von Mises elastoplastic solids with power-law isotropic strain hardening [19]. The plasticity of amorphous silica is much more involved and an attempt to describe its behavior requires additional data to identify a constitutive law with some confidence.

Recently, Perriot et al. [7] provided a map of the local indentation-induced densification in a fused silica sample. The latter was obtained from Raman microspectroscopic mappings [20] on cross-sections of indents. For that purpose, they used the work of Sugiura et al. [21] which correlates the position of the $D_{2}$ line [22] of the Raman Spectrum of silica with the ratio of the sample density to its initial density. They also provided clear evidence for a densification-induced hardening process with diamond-anvil cell (DAC) measurement [23]. Ji et al. [8] have also evidenced such a densification-induced 
hardening in window glass.

These experimental results provide a rich and complex set of data from which to identify a constitutive equation for silica. In this paper a new plastic yield criterion is proposed and its implementation in a finite element software is explained in details. Then, the identification of the material parameters is carried out using instrumented indentation results. Finally the relevance of the new criterion is assessed by comparison with the experimental density maps $[7,24]$.

\section{Constitutive modelling of amorphous silica}

In standard metallic materials, plasticity is pressure-independent [25]. Hence, for such materials, the yield function is often written :

$$
f\left(\sigma_{i j}\right)=q-Y
$$

where $\sigma_{i j}$ denotes the Cauchy stress tensor, $\mathrm{q}$ is the von Mises equivalent stress and $\mathrm{Y}$ is the yield stress. The macroscopic von Mises stress is proportional to the shear stress [26] and is given by :

$$
q=\sqrt{\frac{3}{2} S_{i j} S_{i j}}
$$

In equation (2), $S_{i j}$ is the stress deviator defined by

$$
S_{i j}=\sigma_{i j}+p \delta_{i j}
$$

where $p=-\sigma_{k k} / 3$ is the hydrostatic pressure and $\delta_{i j}$ is the Kronecker tensor. In associative plasticity the plastic rate of deformation is normal to the yield 
surface [27]. This leads to :

$$
\dot{\varepsilon}_{i j}^{p}=\dot{\lambda} \frac{\partial f}{\partial \sigma_{i j}}
$$

where $\dot{\lambda}$ is the plastic multiplier which can be determined from the consistency condition [28], $f\left(\sigma_{i j}\right)=0$ during plastic yielding.

For obvious reasons, such a criterion cannot account for the silica glass densification under pure hydrostatic loading and a relation between $f\left(\sigma_{i j}\right)$ and the hydrostatic pressure $p$ has to be taken into account instead. Recently, Xin et al. $[29,30]$ proposed the following criterion for amorphous silica :

$$
f\left(\sigma_{i j}\right)=\alpha q+(1-\alpha) p-\beta
$$

This pressure-dependent yield criterion resembles the Mohr-Coulomb criterion initially developed for frictional materials [31] and involves two physical characteristics: $i$ ) the yield stress $\beta$; ii) the densification factor $\alpha$ which balances the respective influence of applied hydrostatic pressure, $p$, and von Mises stress, $q$. Under the assumption of an associated perfect-plastic behavior, Xin and Lambropoulos [30] identified these two parameters by comparing experimental load/displacement indentation curves to finite element simulation results. With the values $\alpha=0.6$ and $\beta=9.4 \mathrm{GPa}$, they adequately reproduced their experimental data.

Recently, Perriot et al. [7] used Raman micro-spectroscopy to estimate indentationinduced densification in silica. The micrometric spatial resolution of this technique allowed them to perform a mapping of the permanent densification below an indentation imprint. It was found that the densification increases gradually from 0 to $20 \%$ when approaching the contact area while the Kim model, 
however, predicts a sharp transition between a fully densified hemispherical inclusion and a non densified bulk. Hence the simple criterion (4) needs to be refined to account for these additional experimental results.

Two comments regarding the limitations of such a Mohr-Coulomb like criterion should be made at this stage. First, the linearity of (4) together with the hypothesis of associated flow rule, implies that the direction of the plastic strain rate does not depend on pressure $p$ and shear stress $q$. Moreover, no hardening is taken into account in this constitutive equation. Even if the relevance of hardening in metallic glasses is still a matter of discussion [11], the existence of a hardening effect has been clearly established in the case of silica[7]. To overcome these limitations, we propose in the following a new constitutive equation. We focus first on the yield criterion and then discuss how the hardening can be incorporated.

\subsection{A new yield criterion for fused silica}

A strong dependence on pressure is the main characteristic of the plastic behavior of fused silica. For that reason silica may appear as closer to powders or geomaterials than to metals or crystalline materials. Yield functions designed to account for the compaction of metal powders [32,33] thus exhibit similar dependences with hydrostatic pressure. In the present case, however, there is no evidence of frictional effect and no reason to depart from associative plasticity. Moreover, in the absence of indications of a pressure effect in tension we will ignore it for negative hydrostatic pressure.

To account for the plasticity of fused silica we propose, for positive pressures, 
an elliptic criterion (see Fig. 1) similar to the one used by Shima and Oyane [34] to reproduce experiments on copper. For negative pressure we assume a simple von Mises criterion. In compression $(p>0)$, the proposed yield criterion is:

$$
f\left(\sigma_{i j}\right)=\left(\frac{q}{q_{c}}\right)^{2}+\left(\frac{p}{p_{c}}\right)^{2}-1
$$

while in tension $(p<0)$, one has:

$$
f\left(\sigma_{i j}\right)=q-q_{c}
$$

where $p_{c}$ and $q_{c}$ represent the hydrostatic plastic limit in pure hydrostatic state and the shear limit in pure deviatoric state respectively. From equations (3) and (5), the plastic strain rate (for positive pressure) is then given by :

$$
\dot{\varepsilon}_{i j}^{p}=\dot{\lambda}\left(3 S_{i j}-\frac{2 q_{c}^{2}}{3 p_{c}^{2}} p \delta_{i j}\right)
$$

One observes from (5) that the direction of the plastic strain rate now strongly depends on the hydrostatic pressure $p$. For a pure deviatoric stress state, no plastic densification appears - the plastic strain rate is isochoric - whereas for a pure hydrostatic stress only plastic densification can occur.

Finally, in the absence of experimental data on a potential shear hardening effect, we shall only consider density hardening: as sketched in Fig. 1, $p_{c}$ increases with plastic densification whereas $q_{c}$ remains constant. The density hardening behavior of silica is addressed in the next section. 


\subsection{Densification-induced hydrostatic hardening}

The densification-induced hardening has been measured by Perriot et al. [24] with the help of a Diamond-Anvil Cell apparatus [23,35]. Amorphous silica samples have been submitted to successive loading/unloading cycles of growing maximum pressure $P_{\max }$. At the end of each cycle, a Raman spectrum was measured and the densification was quantified from the shift of the D2 peak [21][7]. The hardening curve obtained by this procedure is presented in Figure 2 .

Note that densification, i.e. the relative volume change can be related to the first invariant of the small strain tensor $\varepsilon_{m}$ which is called mean strain in the present study:

$$
\frac{\Delta V}{V}=\varepsilon_{k k}=3 \varepsilon_{m}
$$

where $\mathrm{V}$ denotes the initial volume. It should also be noted that the small strain assumption has been implicitly applied to describe the densification in fused silica. This hypothesis is expected to be valid for silica since saturation of the densification is reached around $20 \%$ [5].

Strain hardening phenomena occur when materials exhibit plastic deformation. Therefore, the densification-induced hardening should depend on the plastic densification, which leads us to write :

$$
p_{c}=g\left(\varepsilon_{m}^{p}\right)
$$

where $\mathrm{g}$ is a function which describes the evolution of $p_{c}$ with plastic densification. In this paper, it has been assumed that the hardening is isotropic 
and that the hydrostatic hardening does not depend on the deviatoric plastic strain. The mean plastic strain is computed as follows :

$$
\varepsilon_{m}^{p}=\int_{0}^{t}\left|\dot{\varepsilon}_{m}^{p}\right| d \tau
$$

In view of its implementation in a finite element software, the function $g$ has been fitted with a linear relation as displayed in Figure 2. Hence, Eq. (7) reduces to :

$$
p_{c}=\zeta \varepsilon_{m}^{p}+p_{c 0}
$$

where $p_{c 0}=11.5 \mathrm{GPa}$ is the hydrostatic pressure for which the first plastic densification occurs and $\zeta=100 \mathrm{GPa}$ is the hardening slope. Let us note that this value is only slightly smaller than the bulk elastic modulus, which relates the mean strain to the mean stress in linear elasticity, and is about $K=120 \mathrm{GPa}$ for amorphous silica.

For classic metallic solids, where hardening mainly results from dislocation pinning or entanglement, the shear hardening slope is commonly one hundred times lower than the elastic modulus. In the case of amorphous materials, where plasticity is believed to originate from successive local reorganizations[11], there is no equivalent mechanism. A statistical mechanism based on the progressive exhaustion of the most unstable regions has been recently proposed[36]. In the present case of hardening, the progressive approach to the limit density for amorphous silica, equal to the density of the most dense crystalline analog must be taken into account. In a recent study of the plasticity of window glass which is known to exhibit low densification, Ji et al. [8] used a multi-anvil apparatus to measure the bulk plastic modulus for densification of 
window glass. They obtained a value close to $500 \mathrm{GPa}$, i.e. four times higher than the bulk elastic modulus. To be consistent within the elastic-plastic formalism, this result requires a very large increase in the bulk elastic modulus with plastic densification. This also points to some of the difficulties which underlie the measurement and the analysis of the hydrostatic hardening of glasses.

Note finally that Perriot et al. [7] have observed that the zone where the densification saturates is small compared to the contact area. Hence the saturation process will not be really taken into account in this paper.

\section{Finite element modelling of indentation-induced densification in fused silica}

\subsection{Numerical implementation}

The yield criterion developed in the previous section has been implemented

in the finite element software Systus ${ }^{\circledR}$ [37], using a fully implicit backward Euler scheme [38] to perform the stress update.

A stress update algorithm consists of determining at a time $t+\Delta t$, the stress tensor, from the knowledge of the various physical quantities at time $t$ and the strain increment $\Delta \varepsilon_{i j}$. In a fully implicit scheme, the yield condition is enforced at the end of the step, contrary to fully explicit schemes. Hence, a fully implicit scheme is more accurate than an explicit scheme but the implementation is far more complex.

In the constitutive model, the additive decomposition of the strain rate tensor 
into elastic and plastic parts is assumed. The elastic response is thus hypoelastic [26]. In incremental form the constitutive equation is taken to be :

$$
\hat{\sigma}_{i j}=C_{i j k l}\left(\dot{\varepsilon}_{k l}-\dot{\varepsilon}_{k l}^{p}\right)
$$

where $\hat{\sigma}_{i j}$ is a suitable objective stress rate and $C_{i j k l}$ is the fourth rank elastic tensor. Over one step, Eq. (9) can be approximated by:

$$
\Delta \sigma_{i j}=C_{i j k l}\left(\Delta \varepsilon_{k l}-\Delta \varepsilon_{k l}^{p}\right)
$$

Eq. (3) can also be approximated by :

$$
\Delta \varepsilon_{i j}^{p}=\Delta \lambda \frac{\partial f}{\partial \sigma_{i j}}
$$

Substituting Eq. (11) into Eq. (10), and using the consistency condition and Eq. (8) leads to a set of non-linear equations where the unknowns are $\Delta \lambda$ and $\Delta \varepsilon_{m}^{p}$. This set of equations is solved using a Newton scheme [38].

\subsection{Finite element model}

Calculations have been performed using axisymmetric elements to model cone indentation and using a large displacement / large strain option (updated Lagrangian formulation, logarithmic strain). The objective rate of the stress tensor used is the Green-Naghdi rate [38]. The mesh is specially refined near the contact zone. Its spatial extent is large enough to make the results unsensitive to the location of the outer boundaries [39]. The size of the global mesh is one hundred times smaller than the contact radius. For a good representation of the contact geometry, the width of the elements has been determined so 
that at least 40 nodes are in contact for the deepest penetration. The whole mesh contains about 9000 elements and 9400 nodes (see Fig. 3). The contact between the indenter and the workpiece is assumed to be frictionless and loading is achieved by imposing a quasi-static displacement of the indenter which is pushed vertically into the workpiece. The indenter is assumed to be perfectly rigid.

\section{Identification and numerical results}

The constitutive model developed in this paper depends on two main physical quantities $p_{c}$ and $q_{c}$ which represent respectively the plastic limit in a pure hydrostatic state and the shear limit in a pure deviatoric state. The first quantity $p_{c}$ depends on the densification and has been identified from DAC experiments whereas the second $\left(q_{c}\right)$ is constant and unknown. Before being able to confront the results of the finite element simulations with the experimental densification map obtained by Raman micro-spectroscopy, the value of $q_{c}$ has to be identified. Following Xin et al. [30], we used an inverse analysis based on the comparison of experimental load/displacement indentation curves with finite element simulations. For that purpose, five nanoindentation tests on fused silica samples were performed with the help of a Nano-indenter XP (MTS). The maximum penetration depth (about $2 \mu \mathrm{m}$ ) has been chosen large enough so that the influence of indentation length scales [40] on the measured load-penetration curve could be neglected. It has also been verified a posteriori by checking the hardness-penetration curve.

Notice that a Berkovich indenter has been used in this study whereas a Vickers indenter was used by Perriot et al. [7]. As both Vickers and Berkovich indenters 
are usually, as here, replaced by an equivalent cone of semi-apical angle of 70 degrees for load-penetration calculations, this substitution is not prejudicial.

One important result of Perriot et al. [7] is the densification gradient in the bulk of the material induced by indentation testing. The densification gradient induced by the equivalent cone indentation will be approximately the same as obtained with a 3D analysis, except just under the edge of the Vickers indenter. It seems also not wise to try fitting perfectly the densification map of Perriot et al according to the high measurement uncertainities. Therefore an axi-symmetric approximation is sufficient to test this constitutive model.

\subsection{Inverse analysis}

When elastoplastic solids are indented by rigid self similar indenters, the principle of geometric similarity can be applied $[17,39,41]$. This principle [42] states that if two indentations are made by the same geometric shapes, then, whatever their size, the strain $\varepsilon_{i j}$ and stress $\sigma_{i j}$ distributions around the indentation will be geometrically similar. Hence, neglecting indenter tip defect and size effects [40], the loading curve is given by

$$
F=C h^{2}
$$

where $F$ is the load applied on the indenter, $h$ is the penetration depth and $C$ is a function of the mechanical properties [14]. One consequence of this principle is that there is one unique value of $q_{c}$ for which the numerical loading curve matches the experimental loading curve, the other mechanical properties being already known. Nevertheless, $C$ is often difficult to quantify accurately by nanoindentation due to tip deffects and size effects. In this paper we pro- 
pose to base the inverse method on the evaluation of the plastically dissipated energy $W_{p l}$ and the elastically restored energy $W_{e l}$ (see Figure 4) during one indentation cycle [17]. The maximal load used for the Raman mapping [7] being much higher than the load used for the nanoindentation testings, the ratios $W_{p l} / W_{t o t}$ and $W_{e l} / W_{t o t}$ where $W_{t o t}=W_{e l}+W_{p l}$ have been chosen to perform the inverse analysis. These ratios represent the percentage of plastically dissipated energy and elastically restored energy over one indentation cycle. They are theoretically independent on the maximal load applied on the indenter and are thus well suited for this study.

Therefore, finite element simulations have been performed with different values of $q_{c}$. The evolution of $W_{e l} / W_{t o t}$ and $W_{p l} / W_{t o t}$ with $q_{c}$ are displayed in Figure 5. $W_{e l} / W_{t o t}$ increases (respectively $W_{p l} / W_{t o t}$ decreases) linearly with $q_{c}$. This result is not surprising since the higher $q_{c}$, the lower the plastic strain. The experimental values of $W_{p l} / W_{t o t}$ and $W_{e l} / W_{t o t}$ are respectively $0.33 \pm 0.01$ and $0.67 \pm 0.01$. The best fit have been obtained for $q_{c}=6.5 \mathrm{GPa}$. The values of the different parameters for this model are summarized in table 1.

The experimental and numerical load-penetration curves are plotted in Fig. 6. Both loading and unloading curves give a satisfactory agreement with the experimental nanoindentation curve.

\subsection{Densification map}

The densification map obtained with this constitutive model is plotted in Fig. 7. As observed with Raman microspectroscopy [7], the iso-densification lines are concentric. Densification is maximum just under the tip and decreases 
when the distance to the tip increases. Let us note that the maximum densification resulting from the finite element analysis is higher than the maximum densification measured by Perriot et al. It could be explained by the saturation of the densification process which is badly taken into account in the present constitutive model. In spite of that, the densification gradient resulting from the finite element analysis is in very good agreement with the experimental data. It can be considered that the constitutive model developed in this paper allows to reproduce succesfully the indentation-induced densification process of silica.

\section{Conclusion}

We have developed a new constitutive law to model the plastic deformation of amorphous silica. The model takes into account the densification-induced hardening observed under purely hydrostatic loading with DAC experiments. The set of material properties has been identified with an inverse analysis based on the load-penetration curve on a fused silica sample. The resulting indentation-induced densification map obtained with this model is in good agreement with the experimental map obtained by Raman microscopy measurements. Therefore, this constitutive law for the plastic behaviour of amorphous silica accurately accounts for the existing indentation data. Still, one noticeable drawback of this model is that shear hardening is not considered. This shortcoming points out that the densification map and the load-penetration curve are not sufficient to identify the full plastic behavior of amorphous silica. For a more complete constitutive law valid for a wider class of loadings, it is necessary to define new experimental tests capable of providing additional 
data on the shear yielding of such materials. Hence, the shear behavior of silica should be more accurately identified and the role of shear on the densification process could be clarified. For this purpose, we are currently working on the finite element analyses of 3D Berkovich indentation, 3D Vickers indentation and Cube Corner indentation. These results will then be compared to experimental results in order to check the relevance of this constitutive model on other indentation data. Beyond the case of indentation, we are also working on the question of densification under scratch loading [43]. The deformation level in this test is much higher than in indentation experiments. It should thus be possible to observe new phenomena, and perhaps to get a better understanding of the scratch resistance of amorphous silica.

\section{Acknowledgements}

The authors would like to thank A. Perriot, J.M. Bergheau and J.L. Loubet for helpful discussions.

\section{References}

[1] J. Zarzycki. Glasses and the vitreous state. Cambridge University Press, 1991.

[2] F.M. Ernsberger. J. Am. Ceram. Soc. 1968, 51:545

[3] Y.W. Rhee, H.W. Kim, Y. Deng, and B.R. Lawn. J. Am. Ceram. Soc. 2001, $84: 561$

[4] E.W. Taylor. Nature 1949, 163:323

[5] J.C. Lambropoulos, S. Xu, and T. Fang. J. Am. Ceram. Soc. 1996, 79:1441 
[6] S. Yoshida, J.C. Sangleboeuf, and T. Rouxel. J. Mat. Res. 2005, 20:3404

[7] A. Perriot, V. Martinez, L. Grosvalet, Ch. Martinet, B. Champagnon, D. Vandembroucq, and E. Barthel. J. Am. Ceram. Soc. 2006, 89:596

[8] H. Ji, V. Keryvin, T. Rouxel, and T. Hammouda. Scripta Mater. 2006, 55:1159

[9] A.S. Argon. Acta Metall 1979, 27:47

[10] M.L. Falk and J.S. Langer. Phys. Rev. E 1998, 57:7192

[11] C.A. Schuh, T.C. Hufnagel, and U. Ramamurty. Acta Mater. 2007, 55:4067

[12] H. Sugiura and T. Yamadaya. J. Non-Cryst. Solids 1992, 144:151

[13] J.T. Hagan. J. Mat. Sci. 1979, 14:462

[14] J.L. Loubet,J.M. Georges, and G. Meille, Microindentation techniques in Material Science and Engineering . in: P.J. Blau and B.R. Lawn, editors. Philadelphia, Pennsylvania: American Society for Testing and Materials, 1986. p.72.

[15] W-C. Oliver and G. Pharr. J. Mat. Res. 1992, 7:1564

[16] W-C. Oliver and G. Pharr. J. Mat. Res. 2004, 19:3

[17] Y-T. Cheng and C-M. Cheng. Mat. Sci. Eng. R 2004, 44:91

[18] G. Kermouche, J-L Loubet, and J-M. Bergheau. Phil. Mag. A. 2006, 86:5667

[19] J. Alkorta, J.M. Martanez-Esnaola, and J. Gil Sevillano. J. Mat. Res. 2005, 20:432

[20] A. Kailer, K.G. Nickel, and Y.G. Gogotsi. J. Raman Spectrosc. 1999, 30:939

[21] H. Sugiura, R. Ikeda, K. Kondo, and T. Yamadaya. J. App. Phys. 1997, 81:1651

[22] F.L. Galeneer. Solid State Communications 1982, 44:1037 
[23] J.M. Besson and P.P. Pinceaux. Rev. Sci. Instrul. 1979, 50:541

[24] A. Perriot. Nanoindentation de couches minces deposées sur substrat de verre de silice. PhD, Paris 6, 2005.

[25] M.F. Ashby and D.R.H. Jones. Materials. Pergamon press, Oxford, 1980.

[26] G.A. Holzapfel. Non linear solid mechanics - a continuum approach for engineering. Wiley, 2000.

[27] R. Hill. The mathematical theory of plasticity. Oxford Press, 1950.

[28] J. Lemaitre and J-L. Chaboche. Mechanics of Solid Materials. Cambridge University Press, 1996.

[29] A. Shorey, K. Xin, K.H. Chen, and J.C. Lambropoulos. Deformation of fused silica : Nanoindentation and densification. Proc. SPIE, vol 3424, 1998. p.72.

[30] K. Xin and J.C. Lambropoulos. Densification of fused silica : Effects on nanoindentation. Proc. SPIE, vol 4102, 2000. p.112.

[31] A.S. Khan and S. Huang. Continuum Theory of Plasticity. Wiley, 1995.

[32] S. Brown and G. Abou-Chedid. J. Mech. Phys. Solids. 1994, 42:383

[33] J. Cedergen, N.J. Sorensen, and A. Bergmark. Mechanics of Materials 2002, $34: 43$

[34] S. Shima and M. Oyane. Int. J. Mat. Sci. 1978, 18:285

[35] A. Polian and M. Grimsditch. Phys. Rev. B 1990, 41:6086

[36] J.C. Baret, D. Vandembroucq, and S. Roux. Phys. Rev. Lett 2002, 89:195506

[37] Systus/Sysweld. User's manual. ESI Group, 2004.

[38] T. Belytschko, W. Kam Liu, and B. Moran. Non linear Finite Elements for Continua and Structures. Wiley, 2000. 
[39] G. Kermouche, J-L Loubet, and J-M. Bergheau. Mechanics of Materials2007, $39: 24$

[40] W.D. Nix and H. Gao. J. Mech. Phys. Solids. 1998, 46:411

[41] G. Kermouche, J-L Loubet, and J-M. Bergheau. Mechanics of Materials 2008, 40:271

[42] D. Tabor. The hardness of solids. J. App. Phys., pages 145,179, 1971.

[43] G. Kermouche, A.-L. Kaiser, P. Gilles, and J-M. Bergheau. wear 2007, 263:1551 


\section{List of Tables}

1 Mechanical properties of the constitutive model developed in this paper (equations (??) and (??))

\section{List of Figures}

1 The proposed yield criterion for amorphous silica

2 Densification-induced hardening obtained from DAC experiments (extracted from Perriot [24]) and the linear law used in this paper

3 FE mesh used for the modelling of the cone indentation test

4 Definition of the plastically dissipated energy $W_{p l}$ and the elastically restored energy $W_{e l}$ from a typical load-penetration curve

$5 \quad \mathrm{FE}$ analysis of the dependence of $W_{e l} / W_{t o t}$ and $W_{p l} / W_{t o t}$ on shear yield limit $q_{c}$. The experimental values of $W_{p l} / W_{t o t}$ and $W_{\text {el }} / W_{\text {tot }}$ are respectively $0.33 \pm 0.01$ and $0.67 \pm 0.01$.

6 Nanoindentation experimental and numerical load-penetration curves

7 Densification map, -left : experimental results, -right : finite element results 


\begin{tabular}{cc|ccc}
$E(\mathrm{GPa})$ & $\nu$ & $p_{c 0}(\mathrm{GPa})$ & $\zeta(\mathrm{GPa})$ & $q_{c}(\mathrm{GPa})$ \\
\hline 72 & 0,18 & 11,5 & 100 & 6.5
\end{tabular}

Table 1

Mechanical properties of the constitutive model developed in this paper (equations

(5) and (8))

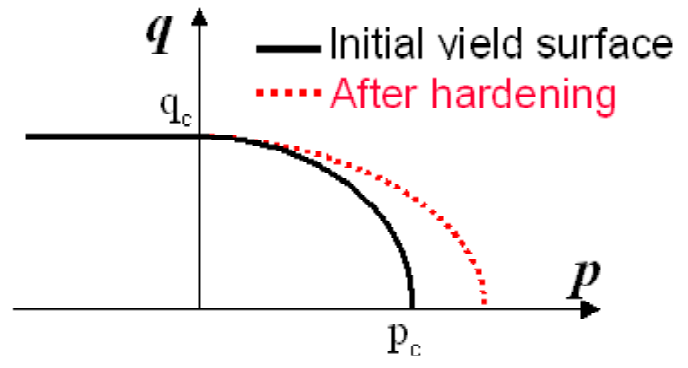

Fig. 1. The proposed yield criterion for amorphous silica 


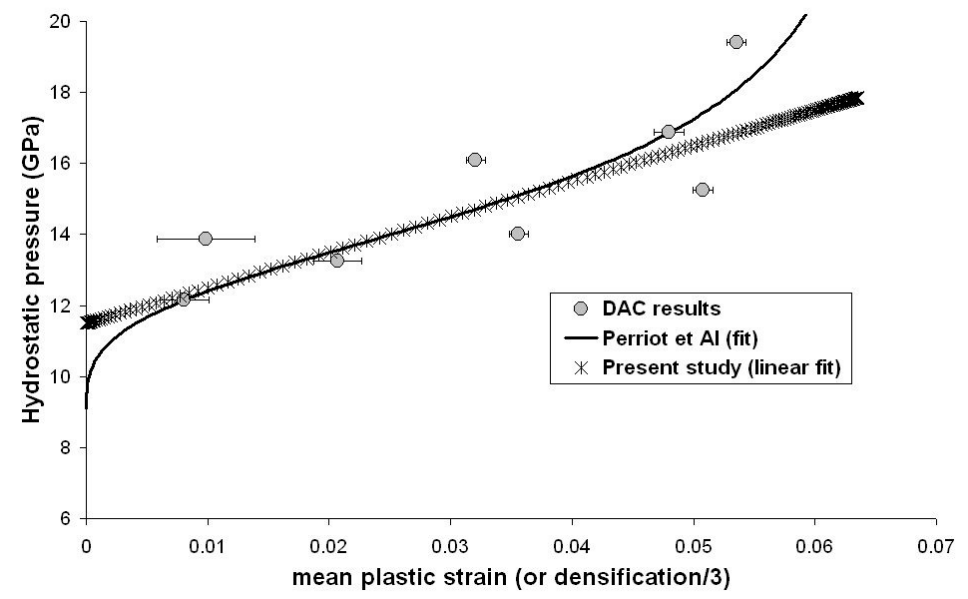

Fig. 2. Densification-induced hardening obtained from DAC experiments (extracted from Perriot [24]) and the linear law used in this paper 


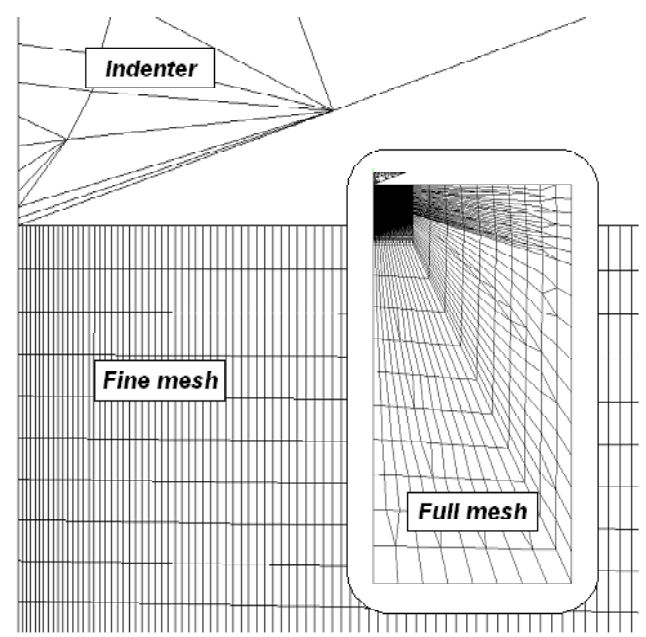

Fig. 3. FE mesh used for the modelling of the cone indentation test

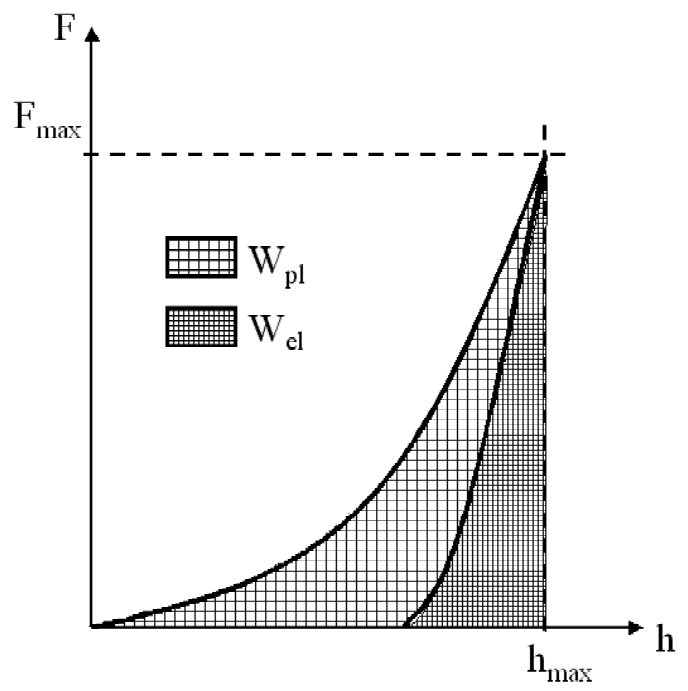

Fig. 4. Definition of the plastically dissipated energy $W_{p l}$ and the elastically restored energy $W_{e l}$ from a typical load-penetration curve 


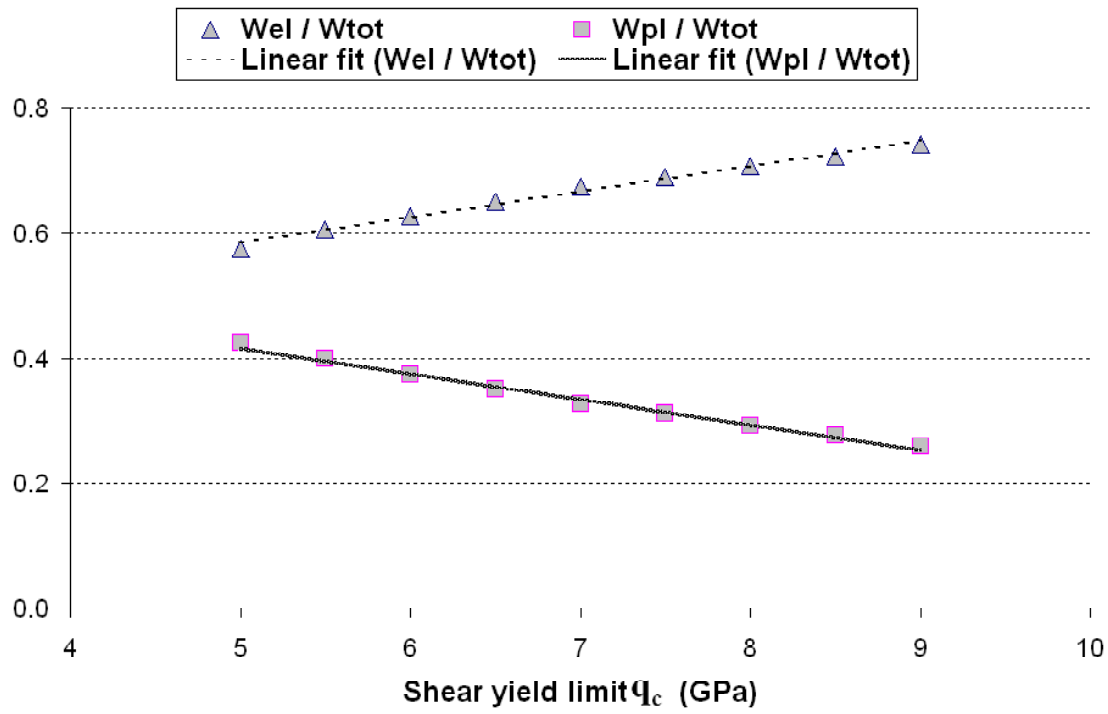

Fig. 5. FE analysis of the dependence of $W_{e l} / W_{t o t}$ and $W_{p l} / W_{t o t}$ on shear yield limit $q_{c}$. The experimental values of $W_{p l} / W_{t o t}$ and $W_{e l} / W_{t o t}$ are respectively $0.33 \pm 0.01$ and $0.67 \pm 0.01$. 


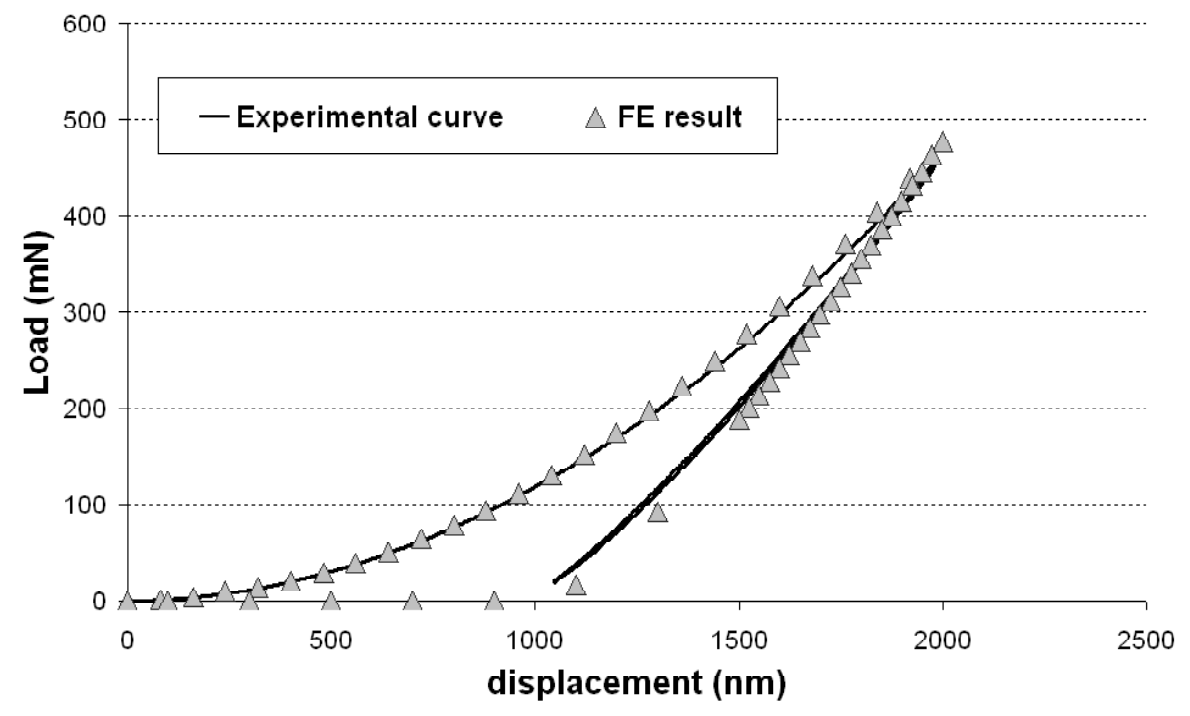

Fig. 6. Nanoindentation experimental and numerical load-penetration curves 


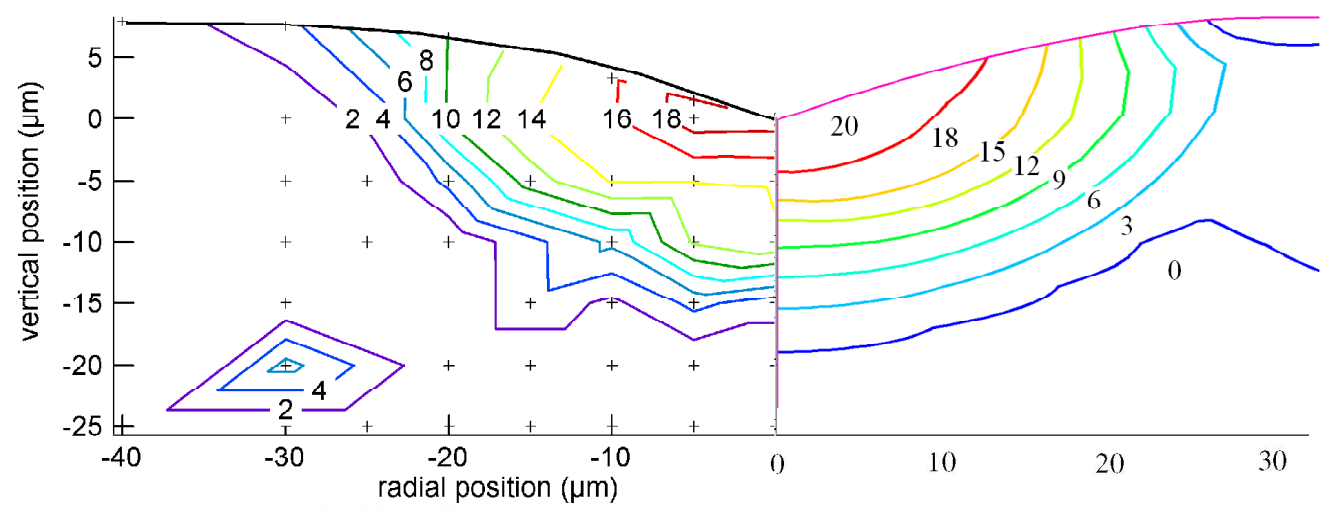

Fig. 7. Densification map, -left : experimental results, -right : finite element results 